\title{
Periodic breathing at high altitude
}

\author{
ANDREW C. FOWLER $†$ \\ Mathematical Institute, University of Oxford, 24-29 St. Giles', Oxford OX1 3LB, UK \\ AND \\ G. P. KALAMANGALAM \\ Department of Neurology, Southern General Hospital, Glasgow G51 4TF, UK
}

[Received on 14 April 2001; revised on 18 December 2002; accepted on 14 January 2003]

\begin{abstract}
Periodic breathing is often associated with heart disease or stroke, and commonly CheyneStokes breathing has a period of about a minute. Periodic breathing also commonly occurs in healthy subjects at high altitude, and here the periods may be much shorter, of the order of 15-20 s. In this paper we study such periodic breathing using the classical model of Grodins et al. (1967, J. Appl. Physiol. 22, 260-276), together with a prescription for the dependence of ventilation on the blood $\mathrm{CO}_{2}$ concentration, modulated by the reduced oxygen pressure (the 'Oxford fan'). The model focusses on the fast dynamics of the arterial blood $\mathrm{CO}_{2}$, and differs in this respect from our previous work which emphasised the brain $\mathrm{CO}_{2}$ concentration; in this sense our model is in fact a generalization of the conceptually simpler Mackey-Glass model.
\end{abstract}

Keywords: Grodins model; periodic breathing; peripheral chemoreceptor; differentialdelay equations.

\section{Introduction}

Periodic breathing (PB) denotes the clinical observation of rhythmic fluctuations of respiration over a timescale of the order of tens of seconds to a few minutes. This is due to (and conversely, causes) corresponding changes in the respiratory blood gases (oxygen and carbon dioxide). Cardiovascular changes (measured, for instance, by blood flow in the major vessels) are a natural accompaniment to $\mathrm{PB}$, due to the physiological interaction of these processes. PB is said to have been recognized by Hippocrates though the modern descriptions are attributed to the Irish physicians John Cheyne and George Stokes in the 19 th century. PB is a feature of severe disease of the heart and/or nervous system, but is also a normal feature in preterm infants, healthy elderly subjects during sleep, and adults taken to high altitude (see Tobin \& Snyder, 1984 or Yamashiro \& Kryger, 1993 for reviews).

The earliest alpine climbers were aware of the effects of altitude on respiration. One of the earliest scientific accounts is given by Mosso (1898), and other early accounts are by Douglas \& Haldane (1909) and, on the effects of altitude, Douglas et al. (1913). A recent account which summarizes much of the early history, and in particular summarizes the achievements of many mountain expeditions, on Everest and elsewhere, is that of Ward et al. (2000).

\footnotetext{
†Email: fowler@maths.ox.ac.uk
} 
The control of respiration over PB timescales is effected by two sets of chemoreceptors, one in the brainstem (that responds exclusively to $\mathrm{CO}_{2}$ ), and the other in the carotid body (that responds to both $\mathrm{O}_{2}$ and $\mathrm{CO}_{2}$ ). Instability in these feedback control loops is thought to underlie the oscillations that constitute PB (Cherniack \& Longobardo, 1986), including that at altitude (Masuyama et al., 1989); this is the conceptual framework for a number of mathematical models (Mackey \& Glass, 1977; Khoo et al., 1982; Carley \& Shannon, 1988; Longobardo et al., 1989; ElHefnawy et al., 1990, Batzel \& Tran 2000a, 2000b, 2000c), including ours (Fowler et al., 1993; Kalamangalam, 1995; Fowler \& Kalamangalam, 2000). The particular focus of our work has been the use of the classical simulation of Grodins et al. (1967) as a base from which to construct (and analyse) reduced, asymptotic mathematical descriptions. We have shown that separation of characteristic timescales identifies the central receptor's role in mediating the slower (time periods of around one minute) forms of $\mathrm{PB}$, due to the dynamics of $\mathrm{CO}_{2}$ distribution. Here we report results of a similar analysis of $\mathrm{CO}_{2}$ dynamics at the peripheral receptor. The influence of $\mathrm{O}_{2}$ is only included through its parametric effect on the $\mathrm{CO}_{2}$ controller, but this is sufficient to predict instability, and also the relatively short oscillation periods associated with hypoxia at high altitude. The recent papers by Batzel \& Tran (2000b, 2000c) have a very similar ethos, but differ from the present work insofar as we identify the rôle of key dimensionless parameters, and are able to derive simplified models on the basis of formal asymptotic approximations.

\section{A reduced Grodins model}

The Grodins model is a compartment model, which describes the evolution of the blood gas concentrations of oxygen and carbon dioxide in three principal compartments: lungs, brain, and other tissues. Grodins et al. (1967) also include the cerebrospinal fluid (CSF) as a further compartment. Figure 1 is a schematic illustration of how the compartments are linked by arterial and venous blood flow. The concentrations in each compartment are described by ordinary differential equations, but the passage time of blood flow between compartments provides (significant) delays, and it is these delays which cause the complexity of the model. They are also responsible for the instability which leads to periodic breathing.

The equations of the Grodins model which concern us are those for $\mathrm{CO}_{2}$ transport, and the three equations (1.1), (1.4) and (1.7) of Grodins et al. (1967) can be written in the approximate form

$$
\begin{gathered}
K_{\mathrm{L}} \dot{P}_{\mathrm{aCO}_{2}}=-\dot{V} P_{\mathrm{aCO}_{2}}+863 K_{\mathrm{CO}_{2}} Q\left[P_{\mathrm{vCO}_{2}}-P_{\mathrm{aCO}_{2}}\right] \\
K_{\mathrm{CO}_{2}} K_{\mathrm{B}} \dot{P}_{\mathrm{BCO}_{2}}=M R_{\mathrm{BCO}_{2}}+K_{\mathrm{CO}_{2}} Q_{\mathrm{B}}\left[P_{\mathrm{aCO}_{2}}\left(t-\tau_{\mathrm{aB}}\right)-P_{\mathrm{BCO}_{2}}\right] \\
-D_{\mathrm{CO}_{2}}\left[P_{\mathrm{BCO}_{2}}-P_{\mathrm{CSFCO}_{2}}\right] \\
K_{\mathrm{CO}_{2} K_{\mathrm{T}} \dot{P}_{\mathrm{TCO}_{2}}=M R_{\mathrm{TCO}_{2}}+\left(Q-Q_{\mathrm{B}}\right) K_{\mathrm{CO}_{2}}\left[P_{\mathrm{aCO}_{2}}\left(t-\tau_{\mathrm{aT}}\right)-P_{\mathrm{TCO}_{2}}\right] .}
\end{gathered}
$$

The meaning of these equations is as follows. $P$ denotes a partial pressure, and the suffix $\mathrm{CO}_{2}$ indicates these are partial pressures of carbon dioxide; then $\dot{P}$ is a time derivative of $P$. However, $\dot{V}$ is not a time derivative as such, but is simply the conventionally used symbol for the ventilation rate, in units of litres per minute. From the mathematical point of view, it can be defined as the local time average of the rate 


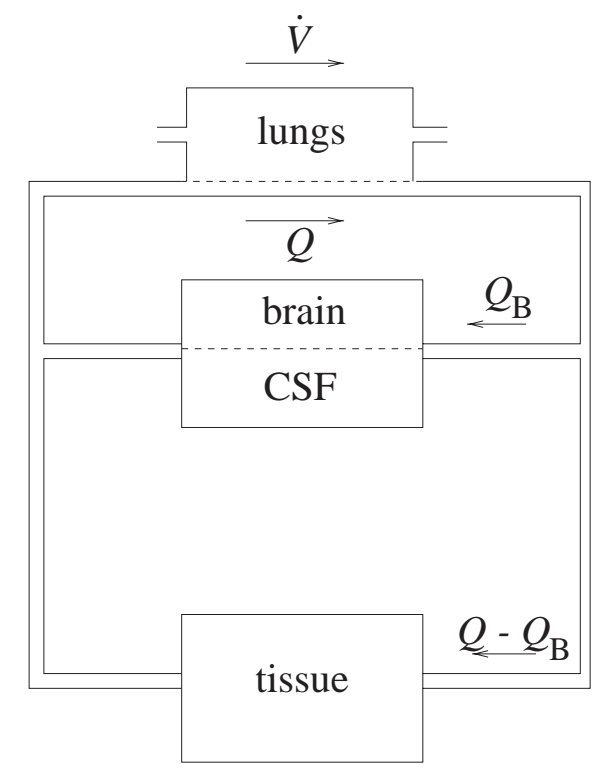

FIG. 1. A schematic illustration of the compartments of the Grodins model. The system is driven by the supply of $\mathrm{O}_{2}$ to the lungs, and by the generation of $\mathrm{CO}_{2}$ within the tissues. The rate of exchange at the alveoli in the lungs is controlled by the ventilation $\dot{V}$.

of inspiration. The other suffixes a, L, B, T, v, and CSF refer to arterial blood, lungs, brain, tissues, venous blood, and cerebrospinal fluid compartments, respectively. In fact, blood gases are measured as concentrations, and these are converted to partial pressures by assuming a locally linear proportionality factor $K_{\mathrm{CO}_{2}}$. The other quantities are $K$, compartment volumes; $M R$, metabolic production rates, and $Q$, blood flow rate; $Q_{\mathrm{B}}$ is the blood flow rate to the brain. The delays $\tau$ represent passage time of blood flow between compartments as indicated by the suffixes; thus $\tau_{\mathrm{aB}}$ represents passage time of arterial blood (from the lungs) to the brain. (In this simple model, the pulmonary circulation is ignored, so that the heart and lungs are located next to each other.) The factor 863 arises in converting inhaled dry gas volumes at standard temperature and pressure (STPD) to body temperature and pressure saturated (BTPS) (West, 1990).

Various assumptions have been made in writing (2.1). In particular, we have equated alveolar partial pressures $P_{\mathrm{A}}$ and arterial partial pressures $P_{\mathrm{a}}$ : this is reasonable. The Grodins model includes an equation for $\mathrm{CSF} \mathrm{CO}_{2}$ concentrations, but we ignore this here since the transport coefficient $D_{\mathrm{CO}_{2}}$ in $(2.1)_{2}$ is small; then (2.1) provides three equations for the four partial pressures: arterial, venous, brain, tissues. They must be supplemented by an equation which represents the partitioning of the blood $\mathrm{CO}_{2}$ load between brain and tissues: this is

$$
Q P_{\mathrm{vCO}}=Q_{\mathrm{B}} P_{\mathrm{BCO}_{2}}\left(t-\tau_{\mathrm{vB}}\right)+\left(Q-Q_{\mathrm{B}}\right) P_{\mathrm{TCO}_{2}}\left(t-\tau_{\mathrm{vT}}\right)
$$

here $\tau_{\mathrm{vB}}$ is the passage time of venous blood from brain back to the lungs, for example. In 
addition, blood flow responds to changes in ventilation, and we will provide a model for such variations later.

\section{Non-dimensionalization}

We begin by writing the model dimensionlessly. Suppose a typical blood flow rate is $Q^{*}$, and a typical flow to the brain is $Q_{\mathrm{B}}^{*}$. Reasonable values are $Q^{*}=61 \mathrm{~min}^{-1}, Q_{\mathrm{B}}^{*}=0.75$ $1 \mathrm{~min}^{-1}$, so that $Q_{\mathrm{B}}^{*} \ll Q^{*}$. We also let $P^{*}$ be a normal alveolar (and thus arterial) $\mathrm{CO}_{2}$ partial pressure, and $\dot{V}_{0}$ be a normal ventilation rate. Such values are $P^{*}=40 \mathrm{~mm} \mathrm{Hg}$ $(\approx 40$ Torr $)$ and $\dot{V}_{0}=51 \mathrm{~min}^{-1}$.

Now it is a matter of observation that $\mathrm{CO}_{2}$ levels in the body do not vary enormously. For example, venous blood returning to the lungs may only have a $\mathrm{CO}_{2}$ partial pressure of $45 \mathrm{~mm} \mathrm{Hg}$. Thus the blood is rather a large reservoir for $\mathrm{CO}_{2}$, with relatively small amounts being permanently exchanged at the tissues and at the lungs. We take advantage of this in non-dimensionalizing the model by writing the partial pressures in the form

$$
\begin{aligned}
P_{\mathrm{aCO}_{2}}=P^{*}\left[1+\varepsilon p_{\mathrm{a}}\right], & P_{\mathrm{BCO}_{2}} & =\frac{M R_{\mathrm{BCO}_{2}}}{K_{\mathrm{CO}_{2}} Q_{\mathrm{B}}^{*}}+P^{*}\left[1+\frac{\varepsilon}{\gamma} p_{\mathrm{B}}\right], \\
P_{\mathrm{TCO}_{2}}=P^{*}\left[1+\varepsilon+\varepsilon p_{\mathrm{T}}\right], & P_{\mathrm{vCO}_{2}} & =P^{*}\left[1+\varepsilon\left(1+p_{\mathrm{v}}\right)\right],
\end{aligned}
$$

where the parameters $\varepsilon$ and $\gamma$ and the partial pressure $P^{*}$ are defined below. In particular, we expect $\varepsilon$ to be relatively small, to reflect the small variation of $\mathrm{CO}_{2}$ in the body. We also define dimensionless blood flows by putting

$$
Q=Q^{*} q, \quad Q_{\mathrm{B}}=Q_{\mathrm{B}}^{*} q_{\mathrm{B}},
$$

and we define the dimensionless ventilation rate $v$

$$
\dot{V}=\dot{V}_{0} v
$$

finally we choose the dimensionless timescale

$$
t \sim \frac{K_{\mathrm{B}}}{Q_{\mathrm{B}}^{*}}
$$

The parameter $P^{*}$ in (2.3) is explicitly taken to be

$$
P^{*}=\frac{863 M R_{\mathrm{TCO}_{2}} Q^{*}}{\left(Q^{*}-Q_{\mathrm{B}}^{*}\right) \dot{V}_{0}} .
$$

The dimensionless form of (2.1) and (2.2) is then

$$
\begin{aligned}
\frac{1}{\Lambda} \dot{p}_{a} & =q\left(1+p_{\mathrm{v}}-p_{\mathrm{a}}\right)-\left(1+\varepsilon p_{\mathrm{a}}\right) v, \\
\dot{p}_{\mathrm{B}} & =\mu\left(1-q_{\mathrm{B}}\right)+q_{\mathrm{B}}\left[\gamma p_{\mathrm{a}}\left(t-\tau_{\mathrm{aB}}^{*}\right)-p_{\mathrm{B}}\right], \\
\dot{p}_{\mathrm{T}} & =s\left[1+\left(\frac{q-\delta q_{\mathrm{B}}}{1-\delta}\right)\left\{p_{\mathrm{a}}\left(t-\tau_{\mathrm{aT}}^{*}\right)-p_{\mathrm{T}}-1\right\}\right], \\
p_{\mathrm{v}} & =p_{\mathrm{T}}\left(t-\tau_{\mathrm{vT}}^{*}\right)+\frac{\delta q_{\mathrm{B}}}{q}\left[c+\frac{p_{\mathrm{B}}\left(t-\tau_{\mathrm{vB}}^{*}\right)}{\gamma}-p_{\mathrm{T}}\left(t-\tau_{\mathrm{vT}}^{*}\right)\right] .
\end{aligned}
$$


TABLE 1 Typical values of the parameters, as given by Grodins et al. (1967) and Batzel \& Tran (2000a).

\begin{tabular}{|c|c|c|c|}
\hline Symbol & Units & \multicolumn{2}{|c|}{ Typical value } \\
\hline & & Grodins & Batzel \& Tran \\
\hline$Q_{\mathrm{B}}$ & $1 \min ^{-1}$ & 0.75 & 0.8 \\
\hline$Q$ & $1 \min ^{-1}$ & 6 & 6 \\
\hline$\dot{\dot{V}}_{0}$ & 1 (BTPS) $\min ^{-1}$ & 5 & 3.6 \\
\hline$K_{\mathrm{L}}$ & 1(BTPS) & 3 & 3.2 \\
\hline$K_{\mathrm{B}}$ & 1 & 1 & 0.9 \\
\hline$K_{\mathrm{T}}$ & 1 & 39 & 14.1 \\
\hline$K_{\mathrm{CO}_{2}}$ & $1(\mathrm{STPD}) 1^{-1} \mathrm{~mm} \mathrm{Hg}^{-1}$ & [0.005] & 0.0057 \\
\hline$M R_{\mathrm{BCO}_{2}}$ & $1(\mathrm{STPD}) \mathrm{min}^{-1}$ & 0.05 & 0.042 \\
\hline$M R_{\mathrm{TCO}_{2}}$ & $1(\mathrm{STPD}) \mathrm{min}^{-1}$ & 0.182 & 0.178 \\
\hline$P^{*}$ & $\mathrm{~mm} \mathrm{Hg}$ & 40 & 40 \\
\hline$D_{\mathrm{CO}_{2}}$ & 1(STPD) $\min ^{-1} \mathrm{~mm} \mathrm{Hg}^{-1}$ & $0.82 \times 10^{-5}$ & - \\
\hline$\tau_{\mathrm{aB}}$ & $\min (\mathrm{sec})$ & $0.18(11)$ & $0.2(12)$ \\
\hline$\tau_{\mathrm{aT}}$ & $\min (\mathrm{sec})$ & 0.32 (19) & $0.32(19)$ \\
\hline$\tau_{\mathrm{vT}}$ & $\min (\mathrm{sec})$ & $0.59(35)$ & $0.42(25)$ \\
\hline$\tau_{\mathrm{vB}}$ & $\min (\mathrm{sec})$ & $0.11(7)$ & - \\
\hline 863 & mm Hg l(BTPS) $1(\mathrm{STPD})^{-1}$ & 863 & \\
\hline$G_{\mathrm{C}}$ & 1(BTPS) $\min ^{-1} \mathrm{~mm} \mathrm{Hg}^{-1}$ & & 1.8 \\
\hline$G_{\mathrm{P}}$ & 1(BTPS) $\min ^{-1} \mathrm{~mm} \mathrm{Hg}^{-1}$ & & 30 \\
\hline$I_{\mathrm{C}}, I_{\mathrm{P}}$ & $\mathrm{mm} \mathrm{Hg}$ & & 37 \\
\hline$\tau_{\mathrm{Q}}$ & $\min (s)$ & $0.1(6)$ & \\
\hline
\end{tabular}

The parameters appearing in these equations are defined, and have typical values (using the parameter values in Table 1) as follows:

$$
\begin{aligned}
\delta & =\frac{Q_{\mathrm{B}}^{*}}{Q^{*}} \approx 0 \cdot 13, \\
\varepsilon & =\frac{\dot{V}_{0}}{863 K_{\mathrm{CO}_{2}} Q^{*}} \approx 0 \cdot 16, \\
\Lambda & =\frac{863 K_{\mathrm{CO}_{2}} Q^{*} K_{\mathrm{B}}}{K_{\mathrm{L}} Q_{\mathrm{B}}^{*}} \approx 11 \cdot 5, \\
\mu & =\frac{M R_{\mathrm{BCO}_{2}} G_{\mathrm{C}}}{K_{\mathrm{CO}_{2}} Q_{\mathrm{B}}^{*} \dot{V}_{0}} \approx 5 \cdot 8, \\
s & =\frac{\left(Q^{*}-Q_{\mathrm{B}}^{*}\right) K_{\mathrm{B}}}{K_{\mathrm{T}} Q_{\mathrm{B}}^{*}} \approx 0 \cdot 18, \\
\gamma & =\frac{G_{\mathrm{C}} P^{*}}{863 K_{\mathrm{CO}_{2}} Q^{*}} \approx 2 \cdot 78, \\
c & =\frac{M R_{\mathrm{BCO}_{2}}}{M R_{\mathrm{TCO}_{2}}}\left(\frac{Q^{*}}{Q_{\mathrm{B}}^{*}}-1\right)-1 \approx 1 .
\end{aligned}
$$

Grodins et al. (1967) give expressions for the delays (their equations (8.10)-(8.13)) which 
are variable, depending on blood flow. In this paper we will take the delays as being constant, although we allow them to depend on blood flow, which is elevated in hypoxic conditions. This anticipates our later suggestion that time-dependent blood flow variation is relatively small. In this case, the dimensionless delays are defined by

$$
\begin{aligned}
& \tau_{\mathrm{aB}}^{*}=\frac{1}{K_{\mathrm{B}}}\left[\frac{\delta V_{\mathrm{aB}}}{q}+\frac{\tilde{V}_{\mathrm{aB}}}{q_{\mathrm{B}}}\right], \\
& \tau_{\mathrm{aT}}^{*}=\frac{1}{K_{\mathrm{B}}}\left[\frac{\delta V_{\mathrm{aT}}}{q}+\frac{\delta \tilde{V}_{\mathrm{aT}}}{q-\delta q_{\mathrm{B}}}\right], \\
& \tau_{\mathrm{vB}}^{*}=\frac{1}{K_{\mathrm{B}}}\left[\frac{V_{\mathrm{vB}}}{q_{\mathrm{B}}}+\frac{\delta \tilde{V}_{\mathrm{vB}}}{q}\right], \\
& \tau_{\mathrm{vT}}^{*}=\frac{1}{K_{\mathrm{B}}}\left[\frac{\delta V_{\mathrm{vT}}}{q-\delta q_{\mathrm{B}}}+\frac{\delta \tilde{V}_{\mathrm{vT}}}{q}\right],
\end{aligned}
$$

where $V_{\mathrm{aB}} \approx 1.0621, \tilde{V}_{\mathrm{aB}} \approx 0.0151, V_{\mathrm{aT}} \approx 1.0621, \tilde{V}_{\mathrm{aT}} \approx 0.7351, V_{\mathrm{vT}} \approx 2.941$, $\tilde{V}_{\mathrm{vT}} \approx 0.1881, V_{\mathrm{vB}} \approx 0.061, \tilde{V}_{\mathrm{vB}} \approx 0.1881$ are blood vessel volumes. The numerical values are those given by Grodins et al. (1967). The previously undefined quantity $G_{\mathrm{C}}$ is defined to be the central controller gain, i.e. a typical value of the rate of change of ventilation with brain $\mathrm{CO}_{2}$ partial pressure, $G_{\mathrm{C}} \sim \mathrm{d} \dot{V}_{0} / \mathrm{d} P_{\mathrm{BCO}_{2}}$. With this choice, the dimensionless ventilation rate $v$ would have an $O(1)$ derivative as a function of $p_{\mathrm{B}}$, in consideration of the central controller. For the peripheral controller, this is not necessarily the case, but it is convenient to use the same scaling so that both central and peripheral control can be framed within the same dimensionless model.

The timescale $K_{\mathrm{B}} / Q_{\mathrm{B}}^{*}$ is about $80 \mathrm{~s}$, comparable to observed periods of Cheyne-Stokes breathing in patients with heart failure, for example, but significantly larger than the $20 \mathrm{~s}$ periods which can be found in hypoxia at altitude (West et al., 1986). We have used this timescale to begin with because it relates directly to our previous work (Fowler et al., 1993; Fowler \& Kalamangalam, 2000), although the presentation here is much more direct and simpler to follow. Previously, noting that $\Lambda \gg 1$, we supposed effectively that $p_{\mathrm{a}}$ would rapidly approach a quasi-steady state related to $v$, and then $p_{\mathrm{B}}$ would evolve over the $80 \mathrm{~s}$ timescale, satisfying a differential delay equation on the assumption that for the central chemoreceptor control located in the brainstem, $v$ can be taken as a function of brain $\mathrm{CO}_{2}$, i.e. $v=v\left(p_{\mathrm{B}}\right)$. This procedure effectively assumes that peripheral chemoreceptor control is stable.

In this paper, we focus on shorter timescales comparable to the transport time from lung to the peripheral chemoreceptor; we will in fact suppose that the central controller response is stable, but we investigate possible instability due to the peripheral chemoreceptor. Therefore, we now rescale time as

$$
t \sim \tau_{\mathrm{PC}}=\frac{\delta V_{\mathrm{aB}}}{K_{\mathrm{B}}},
$$

so that the relevant dimensional timescale is

$$
[t]=\frac{V_{\mathrm{aB}}}{Q^{*}} \approx 11 \mathrm{~s}
$$


The dimensionless model (2.8) can now be written in the form

$$
\begin{aligned}
\lambda \dot{p}_{a} & =q\left(1+p_{\mathrm{v}}-p_{\mathrm{a}}\right)-\left(1+\varepsilon p_{\mathrm{a}}\right) v, \\
\dot{p}_{\mathrm{B}} & =\hat{\mu}\left(1-q_{\mathrm{B}}\right)+\tau_{\mathrm{PC}} q_{\mathrm{B}}\left[\gamma p_{\mathrm{a}}\left(t-\hat{\tau}_{\mathrm{aB}}\right)-p_{\mathrm{B}}\right], \\
\dot{p}_{\mathrm{T}} & =\hat{s}\left[1+\left(\frac{q-\delta q_{\mathrm{B}}}{1-\delta}\right)\left\{p_{\mathrm{a}}\left(t-\hat{\tau}_{\mathrm{aT}}\right)-p_{\mathrm{T}}-1\right\}\right], \\
p_{\mathrm{v}} & =p_{\mathrm{T}}\left(t-\hat{\tau}_{\mathrm{vT}}\right)+\frac{\delta q_{\mathrm{B}}}{q}\left[c+\frac{p_{\mathrm{B}}\left(t-\hat{\tau}_{\mathrm{vB}}\right)}{\gamma}-p_{\mathrm{T}}\left(t-\hat{\tau}_{\mathrm{vT}}\right)\right],
\end{aligned}
$$

where the additional parameters are

$$
\begin{array}{cc}
\lambda=\frac{1}{\Lambda \tau_{\mathrm{PC}}} \sim 0.66, & \tau_{\mathrm{PC}}=\frac{\delta V_{\mathrm{aB}}}{K_{\mathrm{B}}} \sim 0.13, \\
\hat{\mu}=\mu \tau_{\mathrm{PC}} \sim 0.8, & \hat{s}=s \tau_{\mathrm{PC}} \sim 0.023,
\end{array}
$$

and the new dimensionless delays are

$$
\begin{aligned}
& \hat{\tau}_{\mathrm{aB}}=\frac{1}{q}+\frac{\eta_{\mathrm{aB}}}{q_{\mathrm{B}}}, \\
& \hat{\tau}_{\mathrm{aT}}=\frac{\xi_{\mathrm{aT}}}{q}+\frac{\eta_{\mathrm{aT}}}{q-\delta q_{\mathrm{B}}}, \\
& \hat{\tau}_{\mathrm{vB}}=\frac{\xi_{\mathrm{vB}}}{q_{\mathrm{B}}}+\frac{\eta_{\mathrm{vB}}}{q}, \\
& \hat{\tau}_{\mathrm{vT}}=\frac{\xi_{\mathrm{vT}}}{q-\delta q_{\mathrm{B}}}+\frac{\eta_{\mathrm{vT}}}{q},
\end{aligned}
$$

where

$$
\begin{array}{r}
\eta_{\mathrm{aB}}=\frac{\tilde{V}_{\mathrm{aB}}}{\delta V_{\mathrm{aB}}} \approx 0.113, \\
\xi_{\mathrm{aT}}=\frac{V_{\mathrm{aT}}}{V_{\mathrm{aB}}} \approx 1, \quad \eta_{\mathrm{aT}}=\frac{\tilde{V}_{\mathrm{aT}}}{V_{\mathrm{aB}}} \approx 0.692, \\
\xi_{\mathrm{vB}}=\frac{V_{\mathrm{vB}}}{\delta V_{\mathrm{aB}}} \approx 0.452, \quad \eta_{\mathrm{vB}}=\frac{\tilde{V}_{\mathrm{vB}}}{V_{\mathrm{aB}}} \approx 0.177, \\
\xi_{\mathrm{vT}}=\frac{V_{\mathrm{vT}}}{V_{\mathrm{aB}}} \approx 2.768, \quad \eta_{\mathrm{vT}}=\frac{\tilde{V}_{\mathrm{vT}}}{V_{\mathrm{aB}}} \approx 0.177 .
\end{array}
$$

\section{Ventilation controller}

We now consider the formulation for ventilation $(\dot{V})$. Grodins et al. (1967) in fact proposed two forms, their equations (9.1) and (9.2), in which they took $\dot{V}$ to be the sum of two terms, representing respectively the central and peripheral controllers:

$$
\dot{V}=\dot{V}_{\mathrm{C}}+\dot{V}_{\mathrm{P}}
$$


In the first (earlier) form, the central controller $\dot{V}_{\mathrm{C}}$ was assumed to be a (linear) function of hydrogen ion $\mathrm{H}^{+}$concentration and $\mathrm{CO}_{2}$ partial pressure $P_{\mathrm{BCO}_{2}}$ at the brain; in the second form, the brain $\mathrm{H}^{+}$and $\mathrm{CO}_{2}$ concentrations were replaced by CSF $\mathrm{H}^{+}$concentration, or its equivalent partial pressure $P_{\mathrm{CSFCO}_{2}}$ (via the dissociation constant $K_{\mathrm{CO}_{2}}$ ). As we mentioned above, the rate of transfer of $\mathrm{CO}_{2}$ to the CSF is small, and thus the time constant associated with the $\mathrm{CSF}^{+}$concentration is long. Therefore we can ignore the dependence of $\dot{V}$ on $P_{\mathrm{CSFCO}_{2}}$, in consideration of the much shorter timescales of concern here.

The second term $\dot{V}_{\mathrm{P}}$ represents the peripheral controller in the carotid artery, and is taken by Grodins et al. to depend on the blood oxygen partial pressure $P_{\mathrm{aO}_{2}}\left(t-\tau_{\mathrm{a} 0}\right)$ (the delay $\tau_{\mathrm{a} 0}$ is slightly smaller than that to the brainstem). In the second form of the controller, there is an additional dependence on blood $\mathrm{H}^{+}$concentration at the carotid receptor. The $\mathrm{H}^{+}$concentration is related by a buffering relation (Grodins' equation (3.6)) to $\mathrm{CO}_{2}$ concentration, thus this second controller allows $\dot{V}_{\mathrm{P}}$ to depend (additively) on both $\mathrm{O}_{2}$ and $\mathrm{CO}_{2}$ in the blood.

It should be mentioned that Grodins' controller formulations were based on Gray's (1946) 'multiple factor' theory, surmising an additive dependence of ventilation on the respiratory gases at the centre and periphery. It is now accepted that a multiplicative ('Lloyd-Cunningham') interaction of the second two terms is more accurate (Lloyd et al. 1958). In addition, the ventilatory responses to $\mathrm{CO}_{2}$ and $\mathrm{O}_{2}$ are known to interact: hypoxia (low oxygen) increases the dependence (gain) of $\dot{V}$ on $\mathrm{CO}_{2}$, and hypercapnia $\left(\right.$ high $\mathrm{CO}_{2}$ ) increases the ventilatory response to oxygen (Bellville et al. 1979; Cherniack, 1981). These effects are shown in Figs 2(a) and (b), where the resulting family of curves is known to physiologists as the 'Oxford fan'.

Specific forms of the central and peripheral controllers used by Khoo et al. (1982) which take account of these observations are

$$
\begin{aligned}
& \dot{V}_{\mathrm{C}}=G_{\mathrm{C}}\left[P_{\mathrm{BCO}_{2}}-\left(\frac{M R_{\mathrm{BCO}_{2}}}{K_{\mathrm{CO}_{2}} Q_{\mathrm{B}}^{*}}+I_{\mathrm{C}}\right)\right]_{+}, \\
& \dot{V}_{\mathrm{P}}=G_{\mathrm{P}} \exp \left[-0.05 P_{\mathrm{aO}_{2}}\left(t-\tau_{a 0}\right)\right]\left[P_{\mathrm{aCO}_{2}}\left(t-\tau_{a 0}\right)-I_{\mathrm{P}}\right]_{+},
\end{aligned}
$$

and we will use these to motivate our choice of controller below. In these expressions, $[x]_{+}=\max (x, 0), \tau_{a 0}$ represents the delay in transport between lung and carotid body, and $I_{\mathrm{C}}$ and $I_{\mathrm{P}}$ are threshold values for activation of the respective controllers. The odd choice of threshold in the central controller is because $I_{\mathrm{C}}$ is actually a threshold for the arterial $\mathrm{CO}_{2}$ concentration, related by $P_{\mathrm{aCO}_{2}}=P_{\mathrm{BCO}_{2}}-M R_{\mathrm{BCO}_{2}} / K_{\mathrm{CO}_{2}} Q_{\mathrm{B}}^{*}$ in steady-state conditions.

We write $\dot{V}=\dot{V}_{\mathrm{C}}+\dot{V}_{\mathrm{P}}$ in dimensionless form, using (2.3) and (2.5). With the choice of $\tau_{\mathrm{PC}}$ as timescale, this gives

$$
v=\left[v_{0}+p_{\mathrm{B}}\right]_{+}+g\left[r_{\mathrm{PC}}+\gamma p_{\mathrm{a}}(t-\tau)\right]_{+},
$$

where Grodins et al.'s (1967) equation (8.14) leads to

$$
\tau=\frac{1}{q}+\frac{\eta_{0}}{q_{\mathrm{B}}}
$$

in which

$$
\eta_{0}=\frac{\tilde{V}_{a 0}}{\delta V_{\mathrm{aB}}} \approx 0 \cdot 06
$$




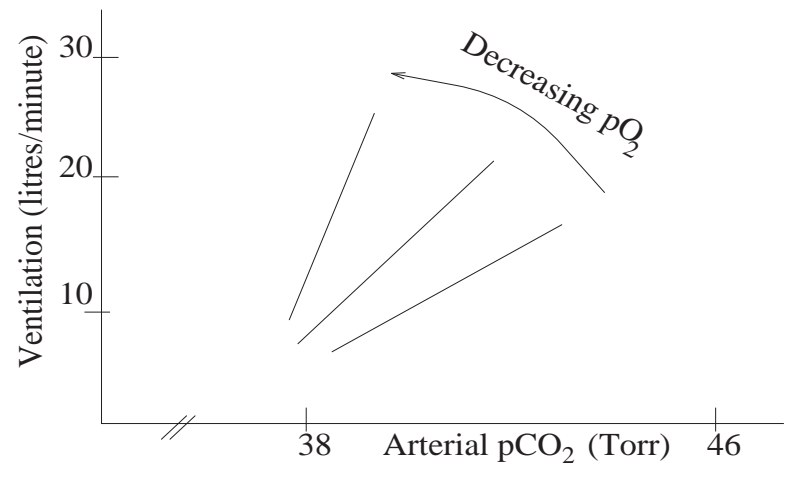

(a)

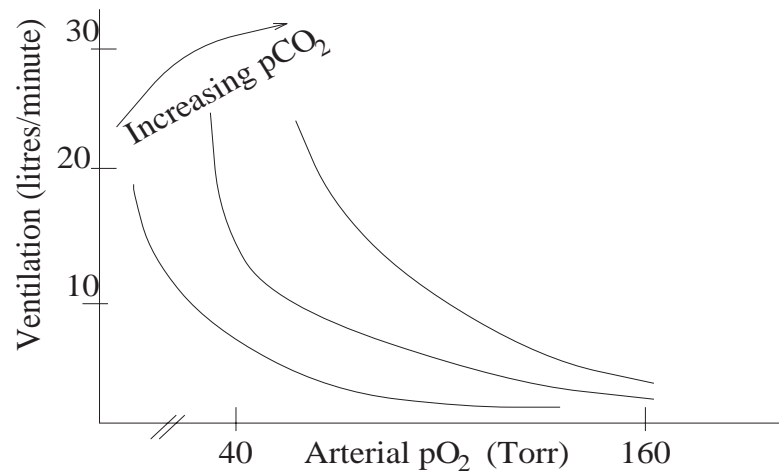

(b)

FIG. 2. (a) The integrated respiratory response to carbon dioxide (dimensional units). Ventilation is an increasing function of $P_{\mathrm{aCO}}$ (carbon dioxide partial pressure), whose slope is perturbed by $P_{\mathrm{aO}}$ (oxygen partial pressure) to produce the 'Oxford fan'. (b) A similar effect of $P_{\mathrm{aCO}}$ on the respiratory response to oxygen. Both figures adapted from Cherniack (1981).

with the Grodins choice $\tilde{V}_{a 0}=0.0081$. We also have

$$
\begin{gathered}
v_{0}=\frac{G_{\mathrm{C}}\left(P^{*}-I_{\mathrm{C}}\right)}{\dot{V}_{0}}, \\
g=\frac{G_{\mathrm{P}} \exp \left[-0.05 P_{\mathrm{aO}_{2}}(t-\tau)\right]}{G_{\mathrm{C}}},
\end{gathered}
$$

and

$$
r_{\mathrm{PC}}=\left(\frac{P^{*}-I_{\mathrm{P}}}{P^{*}-I_{\mathrm{C}}}\right)
$$

If we use the values given in Table 1 , then we find $v_{0}=1.08, r_{\mathrm{PC}}=1$, and $g \approx 0.11,1.4,3.6$ at assumed arterial oxygen partial pressures of $100,50,30 \mathrm{~mm} \mathrm{Hg}$, respectively, the first of these being normal, and the other two representing conditions of hypoxia at high altitude. Evidently the peripheral controller gain responds dramatically to lower oxygen pressure, and it is this effect we wish to study. We do so by focusing on the 
peripheral controller, assuming the response of the central controller is sufficiently slow that it can be considered constant; thus we choose

$$
v=v_{\mathrm{C}}+g\left[1+\gamma p_{\mathrm{a}}(t-\tau)\right]_{+},
$$

where $v_{\mathrm{C}}\left(=\left[v_{0}+p_{\mathrm{B}}\right]_{+}\right)$is the ventilation due to the central controller, and we will consider $g$ to vary parametrically, representing local conditions of hypoxia. The short-timescale variation of oxygen in the blood is not included explicitly. In our numerical solutions, we will use a smoothed form of (2.25), in a similar manner to our previous work (Fowler \& Kalamangalam, 2000). In discussing the values of the parameters, we have simply taken $\dot{V}_{0}$ to be a representative value of the ventilation rate. It is natural to choose $\dot{V}_{0}$ so that, for example, $v_{0}=1$ in (2.22), or so that in steady state, $p_{\mathrm{B}}=0$. The difference is cosmetic, and to be precise, we will define $\dot{V}_{0}$ so that $p_{\mathrm{B}}=0$ in the steady state; equivalently, $p_{\mathrm{a}}=0$ in steady state and thus $P^{*}$ is the steady arterial $\mathrm{CO}_{2}$ partial pressure. The precise definition of $\dot{V}_{0}$ required is given below, in (3.12).

\section{Blood flow}

Grodins et al. (1967) propose two equations describing blood flow. These can be written in the (dimensional) forms

$$
\begin{gathered}
\tau_{\mathrm{Q}} \dot{Q}=U-Q, \\
\tau_{\mathrm{Q}} \dot{Q}_{\mathrm{B}}=U_{\mathrm{B}}-Q_{\mathrm{B}},
\end{gathered}
$$

where the equilibrium blood flow $U$ and brain blood flow $U_{\mathrm{B}}$ are functions of $P_{\mathrm{aO}}$ and $P_{\mathrm{aCO}_{2}}$. Grodins gives complicated algebraic formulae for these, the essence of which is that decreasing arterial oxygen levels below (normal) partial pressure of $100 \mathrm{~mm} \mathrm{Hg}$ cause increased blood flow to the tissues and to the brain, while for arterial $\mathrm{CO}_{2}$ levels above (normal) $40 \mathrm{~mm} \mathrm{Hg}$, blood flow also increases. Blood flow thus behaves similarly to ventilation. Figures 3 and 4 show graphs of the Grodins blood flow curves as a function of $P_{\mathrm{aCO}_{2}}$, under conditions of normoxia and hypoxia. We define dimensionless blood flow functions $u$ and $u_{\mathrm{B}}$ by

$$
U=Q^{*}(\beta-1+u), \quad U_{\mathrm{B}}=Q_{\mathrm{B}}^{*}\left(\beta_{\mathrm{B}}-1+u_{\mathrm{B}}\right),
$$

where $\beta$ and $\beta_{\mathrm{B}}$ are (dimensionless) enhancement factors which increase in hypoxia, and $u$ and $u_{\mathrm{B}}$ are dimensionless $O(1)$ functions of $P_{\mathrm{aCO}_{2}}$, or, when suitably scaled, $p_{\mathrm{a}}$; specifically, $\beta$ and $\beta_{\mathrm{B}}$ are defined so that $u=u_{\mathrm{B}}=0$ at normal $\mathrm{CO}_{2}$ levels, $P^{*}=40$ mm Hg. From Grodins' formulae, we find $\beta=1,1.22,1.56$ for $P_{\mathrm{aO}_{2}}=100,50,30$ $\mathrm{mm} \mathrm{Hg}$ respectively, and $\beta_{\mathrm{B}}=1,1.34,1.79$ for the same three values. Figure 5 shows the dependence of $\beta$ and $\beta_{\mathrm{B}}$ on $P_{\mathrm{aO}_{2}}$ in Grodins' formula.

The blood flow equations can then be written in dimensionless form, using $\tau_{\mathrm{PC}}$ as the timescale, as

$$
\begin{aligned}
v \dot{q} & =\beta-1+u-q, \\
v \dot{q_{\mathrm{B}}} & =\beta_{\mathrm{B}}-1+u_{\mathrm{B}}-q_{\mathrm{B}},
\end{aligned}
$$




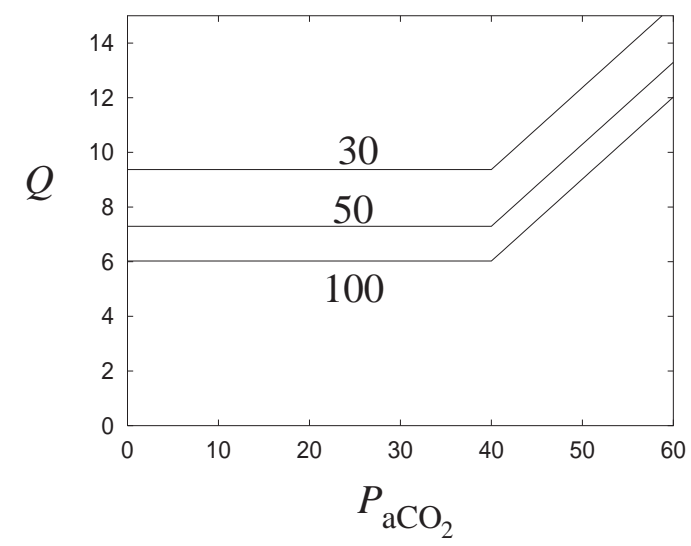

FIG. 3. Blood flow $\left(\mathrm{min}^{-1}\right)$ as a function of $P_{\mathrm{aCO}}(\mathrm{mm} \mathrm{Hg})$ for blood oxygen partial pressures of 100 (normal), $50,30 \mathrm{~mm} \mathrm{Hg}$, according to Grodins et al. (1967).

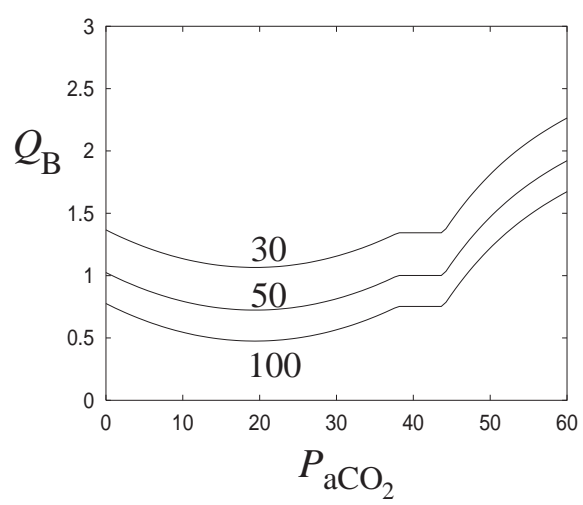

FIG. 4. Blood flow to the brain $\left(1 \mathrm{~min}^{-1}\right)$ as a function of $P_{\mathrm{aCO}}(\mathrm{mm} \mathrm{Hg})$ for blood oxygen partial pressures of 100 (normal), $50,30 \mathrm{~mm} \mathrm{Hg}$, according to Grodins et al. (1967).

where

$$
v=\frac{\tau_{\mathrm{Q}}}{\tau_{\mathrm{PC}}} \approx 0 \cdot 5
$$

One feature of Fig. 3 is that for the small $(O(\varepsilon))$ variations in $P_{\mathrm{aCO}_{2}}$ which occur normally, the variation in the equilibrium blood flow curves is also small. We formalize this by writing

$$
\begin{gathered}
u=1+\varepsilon W\left(p_{\mathrm{a}}\right), \\
u_{\mathrm{B}}=1+\varepsilon W_{\mathrm{B}}\left(p_{\mathrm{a}}\right) .
\end{gathered}
$$

The dimensionless functions $W$ and $W_{\mathrm{B}}$ according to Grodins' formulation are shown in Figs 6 and 7. 


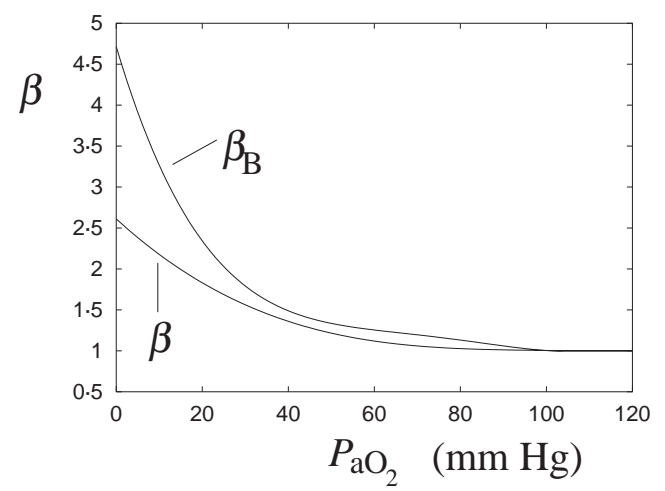

FIG. 5. Enhancement factors $\beta$ and $\beta_{\mathrm{B}}$ as functions of $P_{\mathrm{aO}}$ in $\mathrm{mm} \mathrm{Hg}$.

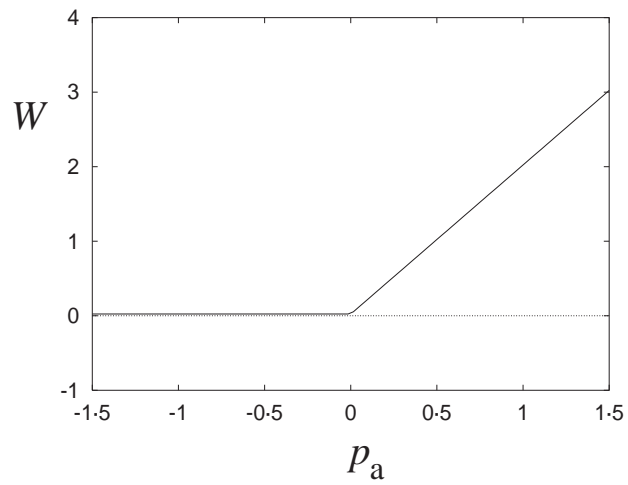

FIG. 6. The dimensionless blood flow function $W\left(p_{\mathrm{a}}\right)$ in (2.30) according to Grodins' formula.

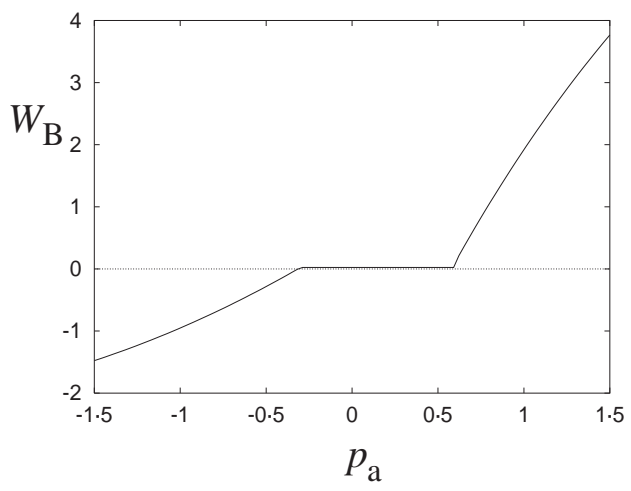

FIG. 7. The dimensionless brain blood flow function $W_{\mathrm{B}}\left(p_{\mathrm{a}}\right)$ in (2.30) according to Grodins' formula. 


\section{Analysis of the model}

The model we wish to study is (2.13) for the gas partial pressures, together with $(2.25)$ for the ventilation, (2.28) with (2.30) for blood flow, and equations such as (2.15) for the delays.

\section{Simplifications}

As it stands, the model requires numerical simulation. However, major simplifications ensue with the use of some judicious approximations. The first of these is that $\delta$ is small, so that if we neglect terms of $O(\delta)$, we have

$$
p_{\mathrm{v}} \approx p_{\mathrm{T}}\left(t-\hat{\tau}_{\mathrm{VT}}\right) .
$$

Next, we observe that $\hat{s}$ is very small. This implies that $p_{\mathrm{T}}$ varies slowly, and then application of the method of averaging to $(2.13)_{3}$ implies that $p_{\mathrm{T}} \approx \bar{p}_{\mathrm{T}}$, its average value, and hence also $p_{\mathrm{v}} \approx p_{\mathrm{T}} \approx \bar{p}_{\mathrm{T}}$; furthermore, $p_{\mathrm{T}}$ tends to a steady state given approximately (for small $\delta$ ) by

$$
1=\overline{q\left(p_{\mathrm{T}}+1-p_{\mathrm{a}}\right)},
$$

where the overbar denotes the time average. Because $p_{\mathrm{v}} \approx p_{\mathrm{T}}$ is slowly varying, $(2.13)_{3}$ can be replaced at leading order in $\delta$ and $\hat{s}$ by

$$
\dot{p}_{v}=\hat{s}\left[1-q\left(1+p_{\mathrm{v}}-p_{\mathrm{a}}\right)\right],
$$

and this can be useful in discussing steady states.

The principal simplification follows if we suppose $\varepsilon$ is small, and we take the blood flow functions as in (2.30); then $q$ and $q_{\mathrm{B}}$ satisfy

$$
\begin{aligned}
v \dot{q} & =\beta+\varepsilon W\left(p_{\mathrm{a}}\right)-q, \\
v \dot{q_{\mathrm{B}}} & =\beta_{\mathrm{B}}+\varepsilon W_{\mathrm{B}}\left(p_{\mathrm{a}}\right)-q_{\mathrm{B}} .
\end{aligned}
$$

If we neglect $O(\varepsilon)$ in these, then we can take blood flow as relatively constant, thus

$$
q=\beta+O(\varepsilon), \quad q_{\mathrm{B}}=\beta_{\mathrm{B}}+O(\varepsilon)
$$

This implies that the delays are approximately constant, and in particular

$$
\tau=\frac{1}{\beta}
$$

since $\eta_{0}$ in (2.21) is small. We also neglect $O(\varepsilon)$ in $(2.13)_{1}$, so that it can be written (using (3.2) together with $\left.p_{\mathrm{v}} \approx p_{\mathrm{T}}\left(t-\hat{\tau}_{\mathrm{vT}}\right) \approx \bar{p}_{\mathrm{T}}\right)$ in either of the equivalent forms

$$
\lambda \dot{p}_{a}=q\left(1+p_{\mathrm{v}}-p_{\mathrm{a}}\right)-v,
$$

or

$$
\lambda \dot{p}=[1+\beta(\bar{p}-p)-v],
$$


where we write $p=p_{\mathrm{a}}$, and the overbar denotes the average. This latter equation reduces the model to a single delay-differential equation for $p$, in which the delay arises through the ventilation, given by $(2.25)$ :

$$
v=v_{\mathrm{C}}+g\left[1+\gamma p_{\tau}\right]_{+},
$$

where $p_{\tau}=p(t-\tau)$. This equation is mathematically analogous to the model of Mackey $\&$ Glass (1977), as we discuss further in the conclusions.

\section{Steady state}

We begin by consideration of the steady state under normal conditions (arterial oxygen of $100 \mathrm{~mm} \mathrm{Hg}$, arterial carbon dioxide of $40 \mathrm{~mm} \mathrm{Hg}$ ). From (2.13), we find that the steadystate partial pressures are given by

$$
p_{\mathrm{a}}=p_{\mathrm{T}}, \quad p_{\mathrm{B}}=\gamma p_{\mathrm{a}}, \quad p_{\mathrm{v}}=p_{\mathrm{T}}+\delta c,
$$

and the steady ventilation rate is

$$
v=\frac{1+\delta c}{1+\varepsilon p_{\mathrm{a}}}
$$

We earlier decided to choose $\dot{V}_{0}$ so that $p_{\mathrm{a}}=p_{\mathrm{B}}=0$ in steady state, thus from (2.19) and (3.11), we finally have

$$
v_{0}=1+\delta c-g r_{\mathrm{PC}},
$$

and this determines (via (2.22)) $\dot{V}_{0}$; specifically,

$$
\dot{V}_{0}=\frac{G_{\mathrm{C}}\left(P^{*}-I_{\mathrm{C}}\right)}{1+\delta c-g r_{\mathrm{PC}}} .
$$

The quantities in (3.13) are independent of $\dot{V}_{0}$ (if we assume $I_{\mathrm{P}}=I_{\mathrm{C}}$ ), except for $P^{*}$, which is given by (2.7), and is inversely proportional to $\dot{V}_{0}$. Thus (3.13) provides an equation for $\dot{V}_{0}$ whose solution is close to the value given in Table 1 .

\section{Steady state at altitude}

Now suppose oxygen partial pressure is reduced. The effect of this is to enhance blood flow, so that $q \approx \beta$ and $q_{\mathrm{B}} \approx \beta_{\mathrm{B}}$. At least while oxygen partial pressure is not too low, $\beta_{\mathrm{B}}-1$ is quite small, so that $p_{\mathrm{B}}$ (via $\left.(2.13)_{2}\right)$ is slowly varying. In fact, even if not, the slow response of $P_{\mathrm{CSFCO}_{2}}$ to $P_{\mathrm{BCO}_{2}}$ implies that the central controller responds to the average $\mathrm{CO}_{2}$ partial pressure. Thus from $(2.13)_{2}$, we have

$$
p_{\mathrm{B}} \approx \gamma \bar{p}-\frac{\mu\left(\beta_{\mathrm{B}}-1\right)}{\beta_{\mathrm{B}}}
$$


brain $\mathrm{CO}_{2}$ is reduced at altitude, and thus the central contribution to ventilation is reduced:

$$
v_{\mathrm{C}} \approx\left[v_{0}+\gamma \bar{p}-\frac{\mu\left(\beta_{\mathrm{B}}-1\right)}{\beta_{\mathrm{B}}}\right]_{+} .
$$

Now we revert to the approximating system in the single equation form (3.8), with ventilation given by (3.9). Clearly from (3.8), the steady state is determined by $v=1$. (In fact there is a mild increase in ventilation at altitude, but this can be understood through the inclusion of $\varepsilon$ in $(2.13)_{1}$, thus $v=1 /\left(1+\varepsilon p_{\mathrm{a}}\right)$, and the increase of ventilation is caused by a decrease of $p_{\mathrm{a}}$ (which we now describe).) From (3.9) and (3.15), the resultant steady arterial $\mathrm{CO}_{2}$ pressure $p=\bar{p}$ is then given by

$$
\left[v_{0}+\gamma \bar{p}-\frac{\mu\left(\beta_{\mathrm{B}}-1\right)}{\beta_{\mathrm{B}}}\right]_{+}+g[1+\gamma \bar{p}]_{+} \approx 1 .
$$

Note that this is inaccurate by $O(\delta)$ at sea level, where $v_{0} \approx 1$ and $g \approx 0 \cdot 1$, because we have neglected the $O(\delta)$ excess of $p_{\mathrm{v}}$ above $p_{\mathrm{T}}$, which implies (via (3.11)) that the steady ventilation rate is actually $1+O(\delta)$.

Since $v_{0} \approx 1$, the central controller is switched off first as $g$ increases at high altitude, and from this it follows that

$$
1+\gamma \bar{p}=\left\{\begin{array}{cl}
\frac{1+g_{\mathrm{c}}}{g_{\mathrm{c}}(1+g)}, & g<g_{\mathrm{c}}, \\
\frac{1}{g}, & g>g_{\mathrm{c}}
\end{array}\right.
$$

where

$$
g_{\mathrm{c}}=\frac{\beta_{\mathrm{B}}}{\mu\left(\beta_{\mathrm{B}}-1\right)},
$$

and we have taken $v_{0}=1$.

As $P_{\mathrm{aO}}$ decreases, the peripheral gain $g$ increases, as does the blood flow to the brain $\beta_{\mathrm{B}}$; hence $g_{\mathrm{c}}$ decreases, and thus the ratio $g / g_{\mathrm{c}}$ increases. Figure 8 shows the dependence of $g / g_{\mathrm{c}}$ on oxygen pressure. From the figure, we have that $g>g_{\mathrm{c}}$ for $P_{\mathrm{aO}_{2}}<59.7$ $\mathrm{mm} \mathrm{Hg}$. At altitudes above the critical height (perhaps around $3000 \mathrm{~m}$ ) where arterial oxygen pressure is about $60 \mathrm{~mm} \mathrm{Hg}$, the theory implies that the central controller fails to provide any ventilatory drive, and ventilation relies solely on the peripheral controller. We now suppose this to be the case.

\section{The underlying map}

It is a further convenience to normalize the delay to be one, by rescaling the time $t \sim \tau$. In this case (3.8) and (3.9) can be written (taking $v_{\mathrm{C}}=0$ ) as

$$
\begin{aligned}
\lambda \dot{p} & =\tau+\bar{p}-p-v^{*}, \\
v^{*} & =g \tau\left[1+\gamma p_{1}\right]_{+},
\end{aligned}
$$




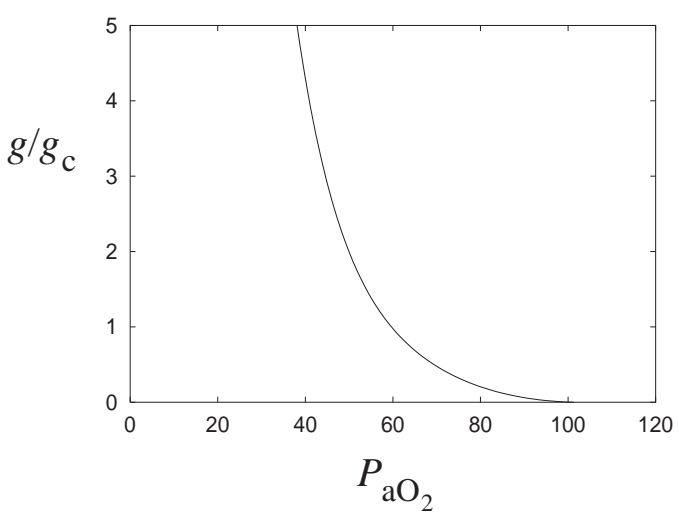

FIG. 8. Variation of $g / g_{\mathrm{c}}$ with arterial oxygen partial pressure ( $\mathrm{mm} \mathrm{Hg}$ ).

since $\tau=1 / \beta$. The system (3.19) takes the form of a delay-recruitment equation,

$$
\lambda \dot{p}=-p+f\left(p_{1}\right),
$$

where

$$
f(p)=\tau+\bar{p}-g \tau[1+\gamma p]_{+} .
$$

It is well known (e.g. Chow et al., 1992) that the dynamics of the differential-delay equation (3.20) are related to the underlying difference map $p=f\left(p_{1}\right)$. In particular, the stability properties of the map are mirrored in the differential-delay equation when $\lambda \neq 0$. Figure 9 shows the effect of decreasing arterial oxygen partial pressure on this map. The slope of the curve at the fixed point $p=\bar{p}$ is $-g \tau \gamma$, so that the fixed point of the map is unstable (to a two cycle) if $g \tau \gamma>1$; as oxygen pressure decreases, $\tau$ decreases but $g$ increases more rapidly, so that $g \tau \gamma$ also increases, and instability occurs for $P_{\mathrm{aO}}<76 \mathrm{~mm} \mathrm{Hg}$. In this situation periodic breathing will occur for sufficiently small $\lambda$, and this will include periods of apnea (i.e. Cheyne-Stokes breathing) if $P_{\mathrm{aO}} \lesssim 60 \mathrm{~mm} \mathrm{Hg}$. Because of the monotonic decreasing form of $f(p)$, there is no further bifurcation as $g$ increases, and a period two cycle of increasing amplitude is the result.

\section{Instability}

The linear stability of the fixed point $\bar{p}$ of (3.21) is studied by seeking values of the exponent $\sigma$ in solutions of the form $p-\bar{p} \propto \exp (\sigma t)$; substituting this into (3.21) and linearizing yields the transcendental equation

$$
\sigma=-\lambda\left[1+\Gamma \mathrm{e}^{-\sigma}\right],
$$

where

$$
\Gamma=g \gamma \tau
$$




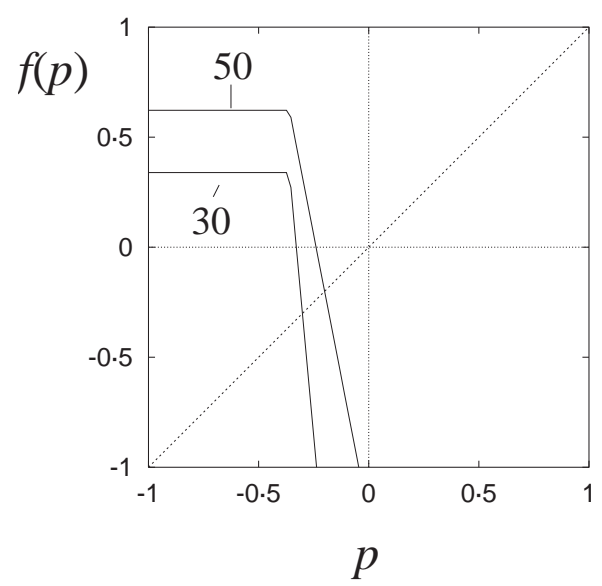

FIG. 9. Form of the underlying map $f(p)$ given by (3.21) for arterial oxygen partial pressures of $50 \mathrm{~mm} \mathrm{Hg}$ and $30 \mathrm{~mm} \mathrm{Hg}$.

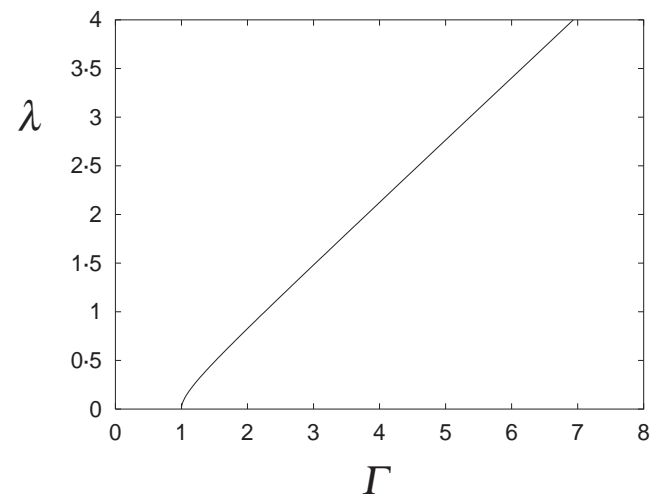

FIG. 10. The linear stability curve of (3.19). The portion to the left and above the curve is stable.

Transition to instability occurs when $\sigma=\mathrm{i} \omega$, which gives the instability curve in $(\Gamma, \lambda)$ space shown in Fig. 10. When $\lambda=0$, instability occurs for $\Gamma>1$, as stated above. For our typical value of $\lambda=0.66$, we have instability for $\Gamma \gtrsim 1.75$, or for $P_{\mathrm{aO}_{2}}<63.8 \mathrm{~mm} \mathrm{Hg}$. There is a small window of arterial oxygen partial pressures for which periodic breathing without apnea occurs, but mostly it will be of Cheyne-Stokes type, with apneic periods.

\section{Numerical simulations}

We have solved (2.13) numerically. The principal result of this is shown in Fig. 11, which compares the linear stability result for the approximating single equation (3.8) with that of the more complicated three-equation model (2.13), and shows that the simpler model gives a good description.

This figure also shows that apneic breathing is the rule for large enough $\Gamma$, or equivalently low enough $P_{\mathrm{aO}_{2}}$; values less than about $60 \mathrm{~mm} \mathrm{Hg}$ give apneic pauses. In 


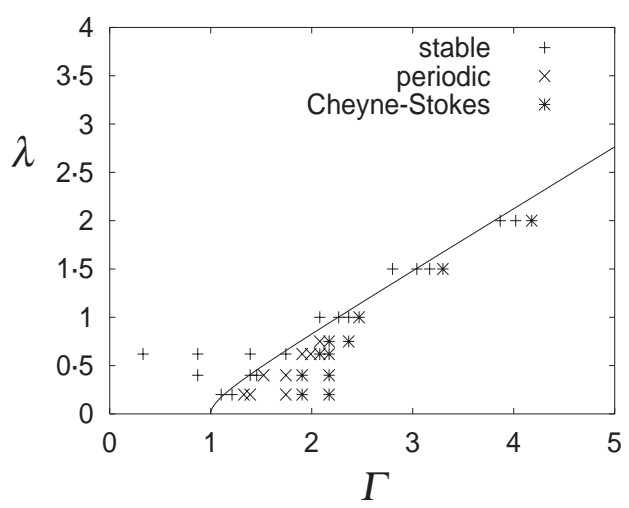

FIG. 11. The stability map of Fig. 10 together with numerical results from solving (2.13). Evidently the stability curve is displaced slightly downwards. Asterisks indicate periodic solutions where apneic periods occurred. A fourth-order Runge-Kutta method was used, with time step 0.01; the piecewise smooth functions in the ventilation controller functions were smoothed with a sharp tanh profile.

the simplified theory, Cheyne-Stokes breathing is predicted for $\Gamma \gtrsim 2 \cdot 7$, or $P_{\mathrm{aO}_{2}}<54$ $\mathrm{mm} \mathrm{Hg}$; this is within the accuracy of the approximations involved.

One can also understand why the apneic/non-apneic transition curve bends around below the stability curve. In the simplified theory, the model is exactly linear until ventilation reaches zero: all unstable oscillations must lead to apnea. However, the neglected term $\varepsilon p_{\mathrm{av}}$ in (2.13) is nonlinear, and allows equilibration of the oscillations without apnea near the stability curve, as we see.

The simplified model also allows us to understand in a qualitative way the length of apneic pauses. Since the model is driven by the requirement that the mean ventilation $\bar{v} \approx 1$, the length of the apneic pauses adjusts itself in order that this be true. Figure 12 shows apneic breathing at $P_{\mathrm{aO}_{2}}=40 \mathrm{~mm} \mathrm{Hg}$ and $\lambda=0.66$. This is deep within the instability region, as $\Gamma=4.87$ at this value. The cycle time is about $24 \mathrm{~s}$, of which about half is in apnea.

West et al. (1986) commented that the linear stability theory of Khoo et al. (1982) underpredicted cycle length at altitudes above about 4000 metres (their Fig. 2). If account is taken of the nonlinear effect of apnea, this is readily understood. At altitude, the average $P_{\mathrm{BCO}_{2}}$ is reduced, following $P_{\mathrm{aO}_{2}}$ down (at $4000 \mathrm{~m}$ altitude, $P_{\mathrm{aO}}$ is in the region of $50 \mathrm{~mm} \mathrm{Hg}$ (Ward et al., 2000)); at sufficient elevation, the central ventilatory control is switched off, and in this situation apnea ensues. The effect of this is to increase the cycle period above that dictated by linear theory, and Fig. 12 indicates that periods of order $20 \mathrm{~s}$ are perfectly possible in this theory.

\section{Conclusions}

Periodic breathing is a common occurrence at altitude, and is often associated with apneic breathing. A particular feature of such breathing is its low period $(\sim 20 \mathrm{~s})$ as compared with the minute long oscillations found in patients with congestive heart failure, often associated with decreased blood flow and consequent increased delay time in transit of blood gases to the central chemopreceptor. 


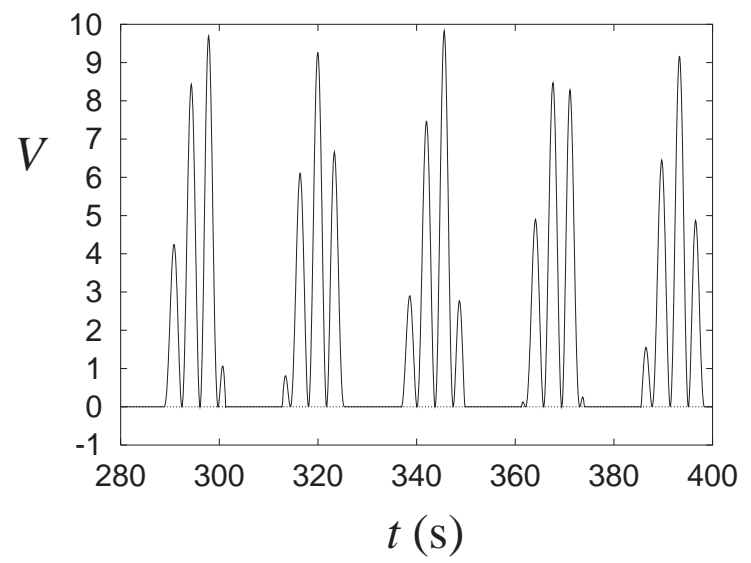

FIG. 12. Apneic breathing at $\Gamma=4.87,\left(P_{\mathrm{aO}_{2}}=40 \mathrm{~mm} \mathrm{Hg}\right)$ and $\lambda=0.66$. The graph of $\dot{V}$ (in dimensionless units) is synthetically modulated by a sine wave to resemble the breath by breath output of a spirogram; specifically, $V=\dot{V}(1+\sin 6 \pi t)$. The horizontal time axis has been converted to dimensional units by multiplication by $[t]=11 \mathrm{~s}$.

In contrast, periodic breathing in hypoxia is associated with the increased gain of the peripheral controller, and we have shown that it can be described by a very simple model describing $\mathrm{CO}_{2}$ fluctuations in the arterial blood, which reduces in its simplest form to a delay-differential equation of delay-recruitment type, similar to the famous model of Mackey \& Glass (1977), although the provenance of the equation is very different. This distinction may be elaborated in future work.

At significant altitudes, the resulting periodic breathing is inevitably apneic, but the period of the cycle (as illustrated in Fig. 12) at higher elevations (thus higher $\Gamma$ ) becomes approximately $2 V_{\mathrm{aB}} / Q^{*}$ (see (2.12)), with half of the cycle being in apnea. This is similar to observed breathing patterns (e.g. West et al., 1986, Fig. 1). Decreasing periods to as low as $15 \mathrm{~s}$ at $8000 \mathrm{~m}$ would be associated in this model with decreasing $V_{\mathrm{aB}} / Q^{*}$, or eqivalently enhanced blood flow. It remains to be seen whether these observations remain valid when oxygen transport is explicitly included in the model, and not just through its effect on the peripheral controller gain, but the resemblance of the simple theory to observation is encouraging.

In this model, the principal parameters controlling instability are $\lambda$ and $\Gamma . \Gamma$ is a complicated function of metabolic $\mathrm{CO}_{2}$ production and ventilatory control parameters, but in a particular individual is primarily a (decreasing) function of arterial oxygen pressure. Thus the horizontal axis in Fig. 12 also represents decreasing $P_{\mathrm{aO}_{2}}$, or increasing altitude. Using (2.9) and (2.11), $\lambda$ is defined by

$$
\lambda=\frac{K_{\mathrm{L}}}{863 K_{\mathrm{CO}_{2}} V_{\mathrm{aB}}} ;
$$

instability is promoted if $\Gamma>1$ (mild elevation) by reduced $\lambda$, for example by reduced effective lung volume. But the essential result is associated directly with increasing altitude. 


\section{Acknowledgements}

This research was supported by a Wellcome Prize Studentship to GPK.

\section{REFERENCES}

An Der Heiden, U. \& Mackey, M. C. (1982) The dynamics of production and destruction: analytic insight into complex behavior. J. Math. Biol., 16, 75-101.

BAtZEL, J. J. \& TRAN, H. T. (2000a) Modeling variable delay and instability in the control system for human respiration: application to infant non-rem sleep. J. Appl. Math. Comput., 110, 1-51.

BAtZEL, J. J. \& TRAN, H. T. (2000b) Stability of the human respiratory control system. I. Analysis of a two-dimensional delay state-space model. J. Math. Biol., 41, 45-79.

BATZEL, J. J. \& TRAN, H. T. (2000c) Stability of the human respiratory control system. II. Analysis of a three-dimensional delay state-space model. J. Math. Biol., 41, 80-102.

Bellville, J. W., Whipp, B. J., Kaufman, R. D., Swanson, G. D., Aqleh, K. H. \& WibERG, D. M. (1979) Central and peripheral loop gain in normal and carotid body-resected subjects. J. Appl. Physiol., 46, 843-853.

CARley, D. W. \& Shannon, D. C. (1988) A minimal mathematical model of human periodic breathing. J. Appl. Physiol., 65, 1400-1409.

Cherniack, N. S. (1981) Respiratory dysrhythmias during sleep. New England J. Med., 305, 325330.

Cherniack, N. S. \& Longobardo, G. S. (1986) Abnormalities in respiratory rhythm. Handbook of Physiology: The Respiratory System Vol. 2. Bethesda, MD: American Physiological Society, pp. 729-749.

Chow, S.-N., Hale, J. K. \& HuAnG, W. (1992) From sine waves to square waves in delay equations. Proc. R. Soc. Edinburgh, 120A, 223-229.

Dahan, A., Degoede, J., Berkenbosch, A. \& Oliver, I. C. (1990) The influence of oxygen on the ventilatory response to carbon dioxide in man. J. Physiol., 428, 485-499.

Douglas, C. G. \& Haldane, J. S. (1909) The causes of periodic or Cheyne-Stokes breathing. J. Physiol., 38, 401-419.

Douglas, C. G., Haldane, J. S., Henderson, Y. \& Schneider, E. C. (1913) Physiological observations made on Pike's Peak, Colorado, with special reference to adaptation at low barometric pressures. Phil. Trans. Roy. Soc. Lond. B, 203, 185-318.

Elhefnawy, A., SAidel, G. M., BruCE, E. N. \& Cherniack, N. S. (1990) Stability analysis of $\mathrm{CO}_{2}$ control of ventilation. J. Appl. Physiol., 69, 498-503.

Fowler, A. C. \& Kalamangalam, G. P. (2000) The role of the central chemoreceptor in causing periodic breathing. IMA J. Math. Appl. Med. Biol., 17, 147-167.

Fowler, A. C., Kalamangalam, G. P. \& Kember, G. (1993) A mathematical analysis of the Grodins model of respiratory control. IMA J. Math. Appl. Med. Biol., 10, 249-280.

GRAY, J. S. (1946) The multiple factor theory of respiratory regulation. Science, 102, 739-744.

Grodins, F. S., Buell, J. \& BART, A. J. (1967) Mathematical analysis and digital simulation of the respiratory control system. J. Appl. Physiol., 22, 260-276.

Kalamangalam, G. P. (1995) Nonlinear oscillations and chaos in chemical cardiorespiratory control. D. Phil. Thesis, University of Oxford.

Khoo, M. C. K., Kronauer, R. E., Strohl, K. P. \& Slutsky, A. S. (1982) Factors inducing periodic breathing in humans: a general model. J. Appl. Physiol., 53, 644-659. 
Lloyd, B. B., Jukes, M. G. M. \& Cunningham, D. J. C. (1958) The relation between alveolar oxygen pressure and respiratory response to carbon dioxide in man. Quart. J. Experim. Physiol., 43, 214-226.

Longobardo, G. S., Gothe, B. \& Cherniack, N. S. (1989) Factors affecting respiratory system stability. Ann. Biomed. Engnr., 17, 377-396.

MACKEY, M. C. \& GLASS, L. (1977) Oscillations and chaos in physiological control systems. Science, 197, 287-289.

Masuyama, S., Kohciyama, S., Shinozaki, T., OKita, S., Kunitomo, F., Tojima, H., Kimura, H., Kuriyama, T. \& Honda, Y. (1989) Periodic breathing at high altitude and ventilatory responses to $\mathrm{O}_{2}$ and $\mathrm{CO}_{2}$. Japan. J. Physiol., 39, 523-535.

Mosso, A. (1898) Life of Man on the High Alps, 2nd edition, (translated Kieso, E. L.). London: Fisher Unwin.

Tobin, M. J. \& SNyder, S. V. (1984) Cheyne-Stokes respiration revisited: Controversies and implications. Critical Care Med., 12, 882-887.

Ward, M. P., Milledge, J. S. \& West, J. B. (2000) High Altitude Medicine and Physiology, 3rd edition. London: Arnold.

West, J. B. (1990) Respiratory Physiology—the Essentials, 4th edition. Baltimore, MD: Williams and Wilkins.

West, J. B., Peters, R. M., Aksnes, G. K. H., Milledge, J. S. \& Schoene, R. B. (1986) Nocturnal periodic breathing at 6300 and 8050m. J. Appl. Physiol., 61, 280-287.

YAmAshiro, Y. \& KRYGer, M. H. (1993) Review: Sleep in heart failure. Sleep, 16, 513-523. 